\title{
Laclias Mexicanas
}

\author{
Por el profesor JUAN BALME, \\ Secretario General de la Sociedad Mexicana \\ "Amigos de las Orquídeas".
}

Entre los numerosos géneros de orquídeas epífitas que tenemos en México, el que corresponde a LAELIA es sin duda alguna el más interesante, no tan sólo por la gran hermosura de las flores que producen diversas especies, algunas de ellas sumamente aromáticas, sino por la gran colección que con las distintas variedades de cada una puede formarse, como lo veremos más adelante en algunas.

Por falta de datos precisos considero imposible señalar el motivo que, para crearlo, en 1831, tuvo el insigne orquidólogo inglés John Lindley, entonces Secretario de la Real Sociedad de Londres y autor de valiosísimas obras sobre orquídeas.

El muy interesante género $L A E L I A$, que mencionó por primera vez en su GENERA ET SPECIES ORCHIDEARUM, según ciertos autores, fué denominado así en memoria del célebre patriota romano CAIUS LAELIUS, renombrado filósofo y orador, conocido por "EL SABIO", cuya esposa e hijas llevaban el apellido LAELIA; otros señalan que, por la gran delicadeza de sus elegantes flores, Lindley lo dedicó a una virgen vestal de mismo nombre.

Teniendo en cuenta la gran erudición del sabio autor, me parece más acertada la primera opinión emitida.

Más tarde, en 1883, dos muy distinguidos botánicos, ingleses también, Bentham y Hooker, cuya clasificación de las orquídeas ha sido y sigue siendo aún a la fecha la más comúnmente aceptada, señalaban en su GENERA PLANTARUM, al género LAELIA y a numerosas especies del mismo, algunas de las cuales, desde el año de 1825, figuraban ya en la valiosa obra de dos de nuestros más distinguidos botánicos del siglo pasado, Juan de la Llave y Pablo Lexarza, pero bajo el nombre genérico de BLETIA. 
En efecto, en el ORCHIDEARUM OPUSCULUM, que en aquella fecha publicaron, figuran las BLETIA grandiflora y autumnalis, especies notables, que se encontraban en gran abundancia en las cercanías de la antigua Valladolid, hoy Morelia, donde aquellos residieron larga temporada:

Estas especies, clasificadas por Lindley con los nombres de LAELIA grandiflora y autumnalis, son las populares "flores de Corpus" y "flores de Todos Santos", que traídas de los bosques cercanos al Distrito Federal, son vendidas en grandes cantidades por las calles de esta Capital, en las épocas respectivas de su floración, durante los meses de abril y mayo las primeras, mientras que las segundas lo son durante los meses de octubre y noviembre.

Es de ese mismo género BLETIA, creado por los botánicos Ruiz y Pavón, y que Reichenbach trató de conservar, que autores más modernos han segregado numerosas especies clasificadas por este último autor, para reunirlas a otros géneros, como son, por ejemplo: BRASSA VOLA, EULOPHIA, HEXALECTRIS, LAELIA y SCHOMBURGKIA, conservando, sin embargo, algunas al mismo género BLETIA, como se verá en la obra que sobre orquídeas mexicanas estoy escribiendo.

Más adelante, al mencionar los sinónimos de cada una, podrán apreciarse aquellos cambios de denominación, aunque sólo señalaré aquellas especies que fueron reunidas al género LAELIA, por ser éste el único que nos interesa en este trabajo.

Como lo saben perfectamente los orqui:dófilos, el género LAELIA, según los autores Bentham y Hooker, pertenece a la tribu de las EPIDENDREAS, en su subtribu de las LELIEAS, pero Schleichter, agregándole otros géneros, que en seguida señalo, ha formado un grupo que designó con el nombre de LAELINAE o LELINEAS, el cual, a más del de las LAELIAS, incluye también los géneros siguientes: BARKERIA, BRASSAVOLA, CATTLEYA, DINEMA, ENCYCLIA, EPIDENDRUM, HOMALOPETALUM, HORMIDIUM, 'MEYRACYLLIUM y SCHOMBURGKIA.

En cuanto a la distribución del género LAELIA, que sólo es de origen americano y del que se cuentan más de 30 especies, es vastísima en todo el Continente, pues lo encontramos desde el N. O. de México, en el Estado de Chihuahua, hasta el S. E. de Brasil, cerca del Uruguay, siendo en nuestro país donde encontramos mayor número de especies, así como de variedades, número que, en parte, podrá apreciarse en la lista que más adelante menciono. 
Fácil es comprender que, con la gran variación de tonos, así como por los vivos colores de matices y máculas, unida a la de tamaños y formas que en cada especie se encuentran, eso ha venido a ser uno de los mayores atractivos que pueda tener el coleccionista, habiéndolos ya, y muy numerosos, quienes sólo se especializan en cultivar LAELIAS mexicanas, por la gran variedad que hay en ellas y sobre todo por su gran rusticidad, pues son, entre las orquídèas epífitas, las más fáciles de cultivar y las que a la vez producen en mayor abundancia muy elegantes flores.

Como se verá en la lista ya nombrada, son ya numerosísimas las variedades y tipos que hay clasificados de aquel género, principalmente en las especies anceps, la más buscada y que cuenta con mayor número de especialistas, quienes la consideran como la más interesante, siendo valiosísima la colección que de ellas hay clasificadas, pero cuantas faltan aún por hacerlo, a más de las 50 que señalo, así como de otras; en particular de las: albida, autumnalis, grandiflora, etc.... tan variadas de formas, tamaños y colores, las que aún abundan en los pocos bosques que nos quedan.

La lista general de especies y variedades de LAELIAS MEXICA$N A S$, sus nombres vulgares y sus sinónimos, que sigue, ha sido tomada de la obra ORQUIDEAS MEXICANAS que estoy terminando y será publicada en el curso de este año.

\section{A E L I A}

acuminata, Ldl., sinónimo de LAELIA rubescens, Ldl.

albida, Batem. Conocida por "monjitas y por tzicxóchitl"

sinónimos: BLETIA albida, Rchb. f.

CATTLEYA albida, Beer.

LAELIA discolor, A. Rich.

variedades:

alba, Hort.

bella, Hort.

majus, Hort.

Mariannae, Hort.

rosea, Hort. sinónimo de bella, Hort.

salmonea, Hort.

sulphurea, Rchb. f. 
superba, Hort.

Stobartii, Hort.

Tuckerii, Hort.

anceps, Ldl. Conocida por "flor de San Miguel" sinónimos: AMALIAS anceps, Hoffmsgg. BLETIA anceps, Rchb. f.

variedades:

CATTLEYA anceps, Beer.

Abekenae, Hort.

alba, Rchb. f.

alba de Bull, Hort.

alba de Worthington, Hort.

amabilis, Hort.

Amesiana, O'Brien.

Ashworthiana, O'Brien.

Ballantineana, Hort.

Barkeriana, Ldl.

sinónimo: LAELIA Barkeriana, Ldl.

blanda, Hort.

Bradshawiana, Hort.

Calvertiana, Hort.

Chamberlainiana, Hort.

sinónimo: grandiflora, Wms.

Crawshayana, Hort.

sinónimo: LAELIA Crawshayana, Rchb. $f$.

Dawsonii, J. Anders.

y varias subvariedades.

delicata, Hort.

Finckeniana, Hort.

sinónimo: LAELIA Finckeniana, Hort.

subvariedad: alba, Hort.

grandiflora, Wms.

Hardyana, Hort.

y subvariedades.

Hollidayana, Hort.

subvariedad: alba, Hort.

holochila, Rolfe.

Hyeana, Hort.

insignis, Hort. 
Kienastii, Hort.

Lageriana, Hort.

Leeana, Rchb. f.

leucosticta, Hort.

major, Hort.

morada, Hort.

munda, Hort.

oculata, Hort.

Percivaliana, Rchb. $\mathrm{f}$.

rosea, Rchb. f.

rubra, Hort.

Sanderiana, Rchb, f.

subvariedad: gigantea, Hort.

Schroederae, Hort.

subvariedades: amesiana, Hort.

Crawshayana, Hort.

Mrs. F. Wellesley, Hort.

Schroederiana, Hort.

Scottiana, Wms.

Simondsii, Hort.

splendens, Hort.

stella, Rchb. f.

Thompsonae, Hort.

vestalis, Hort.

Veitchiana, Rchb. f.

virginalis, Hort. sinónimo: alba, Rchb. f.

Waddonensis, Hort.

aurantiaca, Rolfe.

sinónimo: EPIDENDRUM aurantiacum, Batem.

autumnalis, Ldl. Conocida por "flor de las Animas, flor de Todos

Santos, Ahuaxóchitl y Chichitictepetzacuxóchitl".

sinónimos: BLETIA autumnalis, Llave y Lexarza.

CATTLEYA autumnalis, Beer.

variedades:

alba, Wmis. sinónimo: virginalis, Hort.

atrorubens, Back.

subvariedades.

alba, Hort.

delicatissima, Hort. 
lilacina, Hort.

rosea, Hort.

Fournierii, E. André.

furfuracea, Rolfe, sinónimo: LAELIA furfuracea, Ldl. superba, Hort.

venusta, Goldr., sinónimo: LAELLA venusta, Rolfe. virginalis, Hort., véase alba, Wms.

xanthotropis, Rchb. f.

Barkeriana, Knowl y Westc, véase anceps Barkeriana, Ldl.

Crawshayana, Rchb. f.

Digbyana, Benth y Hooker. Conocida por "flor de plumas blancas". discolor, A. Rich. Véase LAELIA albida, Batem.

erubescens, Du Buys. Véase LAELIA rubescens, Ldl.

Eyermaniana, Rchb. f.

Finckeniana, Hort. Véase LAELIA anceps Finckeniana, Hort. furfuracea, Ldl. Conocida por "lirio de San Francisco". sinónimos: BLETUA furfuracea, Rchb. f. CATTLEYA furfuracea, Beer.

LAELIA autumnalis furfuracea, Rolfe.

variedades:

splendens, Day. sinónimo: LAELIA Gouldiana, Rchb. f. gigantea, Hort., sinónimo: LAELIA grandiflora gigantea, Hort.

glauca, Benth. Conocida por "Candelaria blanca". sinónimo: BRASSAVOLA glauca, Ldl.

Gouldiana, Rchb. f.

sinónimos: LAELIA furfuracea splendens, Day.

variedades:

lilacina, Hort.

purpurea, Hort.

virginale, Hort.

grandiflora, Ldl. Conocida por "flor de mayo, flor de Corpus, Itzmaqua".

sinónimos: BLETIA grandiflora, Llave y Lex.

BLETIA speciosa, H. B. K.

CATTLEYA Grahami, Ldl.

CATTLEYA majalis, Beer.

LAELIA gigantea, Hort.

LAELIA majalis, Ldl.

LAELIA speciosa, Schlecht. 
variedades:

alba, Rchb. f.

atropurpurea, Hort.

delicatissima, Hort.

gigantea, Hort. sinónimo: LAELIA gigantea, Hort.

lilacina, Hort.

majus, Hort.

palida, Hort.

rosea, Hort.

leucoptera, Rolfe.

majalis, Ldl., véase LAELIA grandiflora, Ldl.

peduncularis, Ldl., sinónimo de LAELIA rubescens, Idl.

rubescens, Ldl. Conocida por "flor de Jesús y flor de la Concepción".

sinónimos: BLETIA acuminata, Rchb. b.

BLETIA rubescens, Rchb. f.

CATTLEYA peduncularis, Beer.

LAELIA acuminata, Ldl.

LAELIA erubescens, Du Buys.

LAELIA pcduncularis, Ldl.

variedades:

alba, Hort.

peduncularis, Hook.

rosea, Hort.

speciosa, Schlecht., véase LAELIA grandiflora, Ldl.

superbiens, Ldl. Conocida por "Vara de San José, Candelaria de vara larga y cacho de toro".

sinónimos: BLETIA superbiens, Rchb. f.

CATTLEYA superbiens, Beer.

SCHOMBURGKIA superbiens, Hort.

En la lista anterior sólo están mencionadas las especies tipo, así como los híbridos naturales.

LAELIA gigantea, Hort.

variedades: 
alba, Hort.

decorata, Rchb. f.

grandiflora, Ch. Lem.

Quesneliana, War. y Wms.

Veitchii, Hort., véase LAELIA anceps Veitchiana, Hort. venusta, Rolfe., véase: LAELIA autumnalis venusta, Goldr. 